This document is confidential and is proprietary to the American Chemical Society and its authors. Do not copy or disclose without written permission. If you have received this item in error, notify the sender and delete all copies.

\title{
Computational Investigation on Photohysical Properties of Halogenated Tetraphenyl BODIPY
}

\begin{tabular}{|r|l|}
\hline Journal: & The Journal of Physical Chemistry \\
\hline Manuscript ID & jp-2020-01742w.R1 \\
\hline Danuscript Type: & Article \\
\hline Author: & $\mathrm{n} / \mathrm{a}$ \\
\hline Complete List of Authors: & $\begin{array}{l}\text { Pomogaev, Vladimir; Tomskij gosudarstvennyj universitet, Physics, ; } \\
\text { Kyungpook National University, Department of Chemistry and Green- } \\
\text { Nano Materials Research Center, College of Natural Sciences } \\
\text { Chiodo, Sandro; Universita degli Studi Magna Graecia di Catanzaro, } \\
\text { Dipartimento di Scienze della Salute } \\
\text { Ruud, Kenneth; UiT Norges arktiske universitet, Center for Theoretical } \\
\text { and Cmputational Chemistry } \\
\text { Kuznetsova, Rimma; Tomskij gosudarstvennyj universitet, } \\
\text { Avramov, Pavel; Kyungpook National University College of Natural } \\
\text { Sciences, Chemistry and Green-Nano Materials Research Center }\end{array}$ \\
\hline
\end{tabular}

\section{SCHOLARONE Manuscripts}




\title{
Computational Investigation on Photohysical Properties of Halogenated Tetraphenyl BODIPY
}

\author{
Vladimir Pomogaev ${ }^{\dagger, \square, *}$, Sandro Chiodo ${ }^{\star}, K_{\text {Kenneth Ruud§, Rimma Kuznetsova }}^{\square}$, Pavel \\ Avramov $^{\dagger *}$ \\ 'Department of Chemistry and Green-Nano Materials Research Center, College of Natural \\ Sciences, Kyungpook National University, 80 Daehak-ro, Buk-gu, Daegu, 41566, South Korea. \\ $\square$ National Research Tomsk State University, Lenin Ave. 36, Tomsk 634050, Russia. \\ \$Hylleraas Centre for Quantum Molecular Sciences, Department of Chemistry, University of \\ Tromsø-The Arctic University of Norway, N-9037 Tromsø, Norway. \\ Institut Charles Gerhardt Montpellier, CNRS/ENSCM/UM1/UM2, 8 rue de l'Ecole Normale, \\ 34296 Montpellier Cédex 5, France
}

*Corresponding Author Email: helperv@gmail.com

and paul.veniaminovich@knu.ac.kr 


\section{ABSTRACT}

The electronic structure, transition probabilities and corresponding quantum yields of fluorescence in a family of dihalogen-tetraphenyl-aza-BODIPY were calculated at the TimeDependent Density Functional and post-Hartree-Fock levels of theory. Excellent agreement between theoretical and experimental spectral-luminescent data was achieved with the HSE06 functional and the $6-311 \mathrm{G}^{*}$ basis set. Since the fluorescence can be quenched through nonradiative intersystem spin crossing transitions from the lowest photoactive singlet state to triplet excited states, spin-orbit coupling matrix elements were calculated and applied along with MarcusLevich-Jortner theory, leading to satisfactory agreement for the lifetimes in comparison with available experimental data. The anomalous dependence of the fluorescence efficiency on the atomic number of the halogen congeners was elucidated and shown to be due to an inversion between the fluorescent and the nearest triplet states in the iodinated compounds. The high rate of fluorescence quenching by intersystem crossings and the probability of collisions in a solvent between oxygen molecules and the molecules studied, shows that these molecules can provide efficient triplet sensitization. The most preferable sites for such interactions were predicted using electrostatic potential mapping at the extreme positive and negative charge points.

\section{INTRODUCTION}

The class of alkyl-derivatives of dipyrrolilmethene chromophores (dipyrromethenes, dipyrrines or abbreviation "dpm" when part of larger compounds), in particular complexes with a boron center terminated by $\mathrm{F}_{2}$ (difluoroborates) and known as 4,4-difluoro-4-bora-3a,4a-diaza-s- 
indacene (boron-dipyrromethene, BODIPY, BDP), was synthesized at the turn of the 21 st century. These compounds were originally used as active laser media for liquid and solid-state lasers because of their high extinction coefficients with a radiation efficiency of about 40-60\%, sharp fluorescence bands in the visible region and their extraordinary high photo-stability, which show almost no dependence on the environment. The relatively simple synthesis, high redox and thermodynamic stability along with their unique photophysical properties allowed the complexes to supplant classic laser dyes such as rhodamine, coumarines, oxazinone and even oxazoline dyes. ${ }^{1-4}$

During the last decade, new functional groups were proposed as substituents in these dyes in the development of novel compounds for other technological and biomedical applications. Currently, BDP and its meso-aza derivatives ( $a \mathrm{BDP})$ are synthesized for applications in electrooptical devices and luminescence switches, tunable laser dyes and solid-state solar cells, sensory media for oxygen concentration in gas mixtures and biological fluorescent $\mathrm{pH}$ probes. Particular attention is paid to design chromophore elements for promising ${ }^{1} \mathrm{O}_{2}$ photosensitizers in photodynamic therapy, where energy transfers between the excited triplet sensitizer and groundstate triplet molecular oxygen $\left({ }^{3} \mathrm{O}_{2}\right)$ play an important role. ${ }^{5-7}$ Experimental investigations complemented by computational studies can lead to a deeper understanding of electronic structure, pathways of electronic transitions, and specific spectral-luminescence features, allowing more efficient fluorescent probes, phosphorescent optical sensors and triplet photosensitizers to be developed.

BDPs usually exhibit high fluorescence quantum yields with no significant population of electronic triplet states. However, the inclusion of heavy halogens such as $\mathrm{Cl}, \mathrm{Br}$ or I atoms into the $a \mathrm{BDP}$ core leads to significant nonradiative intersystem spin crossing (ISC) between the lowest 
excited photoactive singlet $S_{1}$ and lower-lying triplet $T_{i}$ states due to an increasing spin-orbit coupling (SOC) with increasing atomic number of the halogen. ${ }^{8}$ In particular, halogenated $a \mathrm{BDPs}$ with different halogen atoms at the edges of the pyrrole rings along the longest molecular axis were tested for their efficacy in destroying tumor cells. ${ }^{9}$

Halogenated BDPs and in particular $a$ BDPs were investigated quantitatively by spectroscopic measurements and by computation to analyze the fluorescence quenching by triplet sensitization. ${ }^{7-}$ 16 Theoretically, a higher efficiency of $S_{1} \rightarrow T_{i}$ transitions should be observed in the iodinated molecule (I- $a \mathrm{BDP})$ than in brominated $a \mathrm{BDP}$ (Br- $a \mathrm{BDP})$ due to stronger SOC and more efficient ISC for "gravity" effect of the heavier halogen congeners. However, this has not observed experimentally. 7,13,14,16,17 Instead, the opposite trend was observed when comparing di-iodine and di-bromine substituted compounds, where $\mathrm{I}_{2}$-tetraphenyl- $a \mathrm{BDP}$ exhibits a quite high quantum fluorescence of about $\gamma=0.33$, whereas $\mathrm{Br}_{2}$-tetraphenyl- $a \mathrm{BDP}$ has almost negligible fluorescence with $\gamma=0.03$ and $\mathrm{Cl}_{2}$-tetraphenyl- $a \mathrm{BDP}$ again appears to display a smaller rate of ISC, with $\gamma=0.28$. These experimental results agreed well with the observed generation of photosensitized $\left({ }^{1} \mathrm{O}_{2}\right)$ by these molecules, where the most effective sensitization was not for the $\mathrm{I}_{2}$-substituted compound, having a triplet yield of 0.12 , but rather for the $\mathrm{Br}_{2}$-substituted sensitizer (triplet yield of 0.83 0.89). ${ }^{7,17}$ Previous computational work have not been able to explain this anomalous $\mathrm{I} / \mathrm{Br}$ sensitization.

Furthermore, systematic shifts in theoretical Time-Dependent Density Functional Theory (TDDFT $)^{18-20}$ fluorescence wavelengths of up to $100 \mathrm{~nm}$ compared to experiment have been reported for the family of dihalogen-tetraphenyl-aza-BDP compounds (XPhaBDP, where $\mathrm{X}=\mathrm{Cl}$, Br, I and $\mathrm{Ph}=$ phenyl substituents). ${ }^{21}$ Satisfactory agreement with experiment for the fluorescence wavelength has only been possible for other BDP derivatives by combining TD-DFT calculations 
with measured crystallographic structures, ${ }^{22}$ and applying the highly accurate and sophisticated multireference complete active space second-order perturbation theory (CASPT2) method. This latter approach has provided calculated results that differ compared with experiment only by about $20 \mathrm{~nm}^{23}$

SOC matrix elements (ME) were calculated for the dibromine-tetramethyl-BDPs at their equilibrium geometry of the electronic ground states, and it was demonstrated that even secondorder coupled-cluster (CC2) gives the lowest excitation energy at $456 \mathrm{~nm}$, substantially different from the experimental wavelength of $540 \mathrm{~nm}$. In contrast, extended multi-configuration quasidegenerate CASSCF/XMCQDPT2 results computed with the Firefly code overestimate the wavelength, giving $579 \mathrm{~nm} .{ }^{15}$ The lowest excited states and the corresponding SOC-ME of several slightly different halogen-derived tetraphenyl-BDPs were studied using TD-DFT combined with pseudopotentials ${ }^{12}$ as implemented in the DALTON code. It was found that the theoretical excitation wavelengths differ by $30-60 \mathrm{~nm}$ compared to experiment. The ISC rates of the $\mathrm{ClPh} a \mathrm{BDP}$ and $\mathrm{BrPh} a \mathrm{BDP}$ were compared assuming weak SOC for which a perturbation theory approach can be used, an assumption that works well for light elements ${ }^{11,24}$ but may be less suited for larger SOC, as can be expected for the heavier iodine atom.

In order to estimate and compare ISC rates of XPh $a$ BDPs, accurate energies of the fluorescent state $S_{1}$ and the lower triplet levels $T_{i}$, as well as $S_{1} \rightarrow T_{i}$ SOC-ME are needed. A careful benchmarking of the quality of different exchange-correlation (XC) DFT functionals ${ }^{25-28}$ with different basis sets is required, both for the emission wavelengths and to establish methods capable of accurately calculating the SOC-ME. This is a necessary prerequisite in order to be able to calculate reliable ISC rates. 
The remainder of the paper is organized as follows: The computational approach is described first. The conformational models of the dipyrrin compounds are discussed next before proceeding to a more detailed description of the electronic structure of the most important conformations. Calculations and analysis of SOC in different compounds, their role in determining the luminescence properties of the molecules and in the interactions with molecular oxygen are then presented. Important details of the computations and comparisons are described in the supplementary information. Some concluding remarks and an outlook are available at the end of the article.

\section{COMPUTATIONAL DETAILS}

A variety of hybrid (PBE029, B3LYP $30,31, \mathrm{M} 062 \mathrm{X}^{32}$ ) and range-separated (HSE06 ${ }^{33,34}$, $\omega B 97 X D^{35,36}$, CAM-B3LYP ${ }^{37}$ ) XC functionals implemented in Gaussian 09 (G09) ${ }^{38}$ were tested using different basis sets (Pople split-valence diffuse 6-311G*(X)/3-21G(H)/6-31G* (6-311G(d)), the split-valence plus polarization basis set (SVP) of Ahlrichs with coworkers ${ }^{39}$ and Dunning's correlation consistent double-zeta basis sets (cc-pVDZ) ${ }^{40}$ to obtain the best agreement with measured spectral-luminescence properties. Different larger versions of the basis sets developed by the Karlsruhe group were also tested: def2-SVP, DZVP, TZVPall and the composite def2TZVPP(X)/def2-SVP(H)/def2-TZVP (dTZVPP), where the notation for the composite basis indicates the basis used for hydrogen $(\mathrm{H}), \mathrm{X}=\mathrm{Cl}, \mathrm{Br}$, I, and then the basis used for the remaining elements.

Ground-state geometries were optimized with the $6-311 \mathrm{G}(\mathrm{d})$ basis sets ${ }^{41}$ both using the HSE06 functional and second-order Møller-Plesset perturbation theory (MP2). ${ }^{42}$ Structural optimizations 
of the lowest photoactive electronic states were carried out using HSE06 combined with the composite DZVP/SVP(H) and 6-311G(d) basis sets. Electronic excitations were calculated using all the varieties of TD-DFT functionals and basis sets mentioned above, as well as both the CC2 method with the resolution of the identity approximation (RI-CC2) ${ }^{43}$ and the second-order algebraic-diagrammatic construction $\operatorname{ADC}(2)^{44,45}$ with the $6-311 \mathrm{G}(\mathrm{d})$ and the dTZVPP basis sets as implemented in the Turbomole 7.2 package (TM7.2). ${ }^{46}$

SOC-ME's were calculated using TD-DFT as implemented in the ADF ${ }^{47}$ and Dalton ${ }^{48}$ packages as well as the stand-alone MolSOC ${ }^{49-51}$ code, which uses KS-DFT results obtained using either Turbomole or Gaussian. Several functionals and basis sets were tested in order to obtain reliable ISC values. SOC-ME's are calculated correctly only using all-electron basis sets, and were limited to the Pople basis sets and the DZVP, TZVPall and SVP basis sets as these were the only all-electron basis sets available for iodine. Dalton, which can calculate SOC-ME for ISC at the TD-DFT level of theory, does not provide the HSE06 functional. For this reason, the most extensive results for the SOC-ME were obtained using the HSE06 functional as implemented in ADF with the Slater-type orbital (STO) basis of TZP and TZ2P quality.

A new release of the MolSOC ${ }^{51}$ code was used to calculate the SOC-ME between the singlet and triplet states using basis sets that included up to $f$ basis functions, following the protocol described in Ref. 50. In all calculations, the one-electron spin-obit operator with effective nuclear charges $\left(\mathrm{Z}_{\mathrm{eff}}\right){ }^{49}$ was used. The procedure provides the signed SOC-ME of single-determinant wave functions for each singlet and triplet transition. To avoid consideration of the full set of singlet$\left(\mathrm{C}_{\mathrm{il}}\right)$ and triplet- $\left(\mathrm{C}^{\mathrm{T}}{ }_{\mathrm{jm}}\right)$ weighted coefficients, which were obtained based on Gaussian and Turbomole electronic structure calculations, a selection procedure using a convergence criterion of $5 \times 10^{-6}$ for the square of the coefficients was used. This approximation was tested by comparing 
with the SOC-ME results obtained with a full set of coefficients, leading to an error of at most $10^{-2}$ $\mathrm{cm}^{-1}$.

\section{RESULTS AND DISSCUSSION}

Spatial XRmBDP structures and symmetries. This paper focuses on the main features of the electronic structure and photophysical properties of a dihalogenated BDP family, abbreviated as $\mathrm{XR} m \mathrm{BDP}$ ( $\mathrm{R}=\mathrm{Phenyl}$, Methyl, $\mathrm{H} ; m=c, a$ which are $\mathrm{C}, \mathrm{N}$ at $m e s o$-position) and, particularly, dihalogen-tetraphenyl-aza-BDP. Atomic numbering follows a symmetric manner (Figure 1) for the 4,4'-dihalogen-3,3',5,5'-tetraphenyl-2,2'-aza-BDP compounds, which differs from the recommended IUPAC rules, but this was chosen to simplify the discussion and presentation. The low-lying excited states of XR $m$ BDPs, the nature of the electron density (ED) redistribution (EDR) as represented by electron density differences (EDD) as well as the rates of fluorescence and radiationless dissipative transitions were analyzed in order to understand photophysical properties of these compounds. A map of the electrostatic potentials (ESP) was produced to estimate chemoand photo-stabilities against anion and/or cation attacks and the interaction with oxygen molecules in collision complexes.

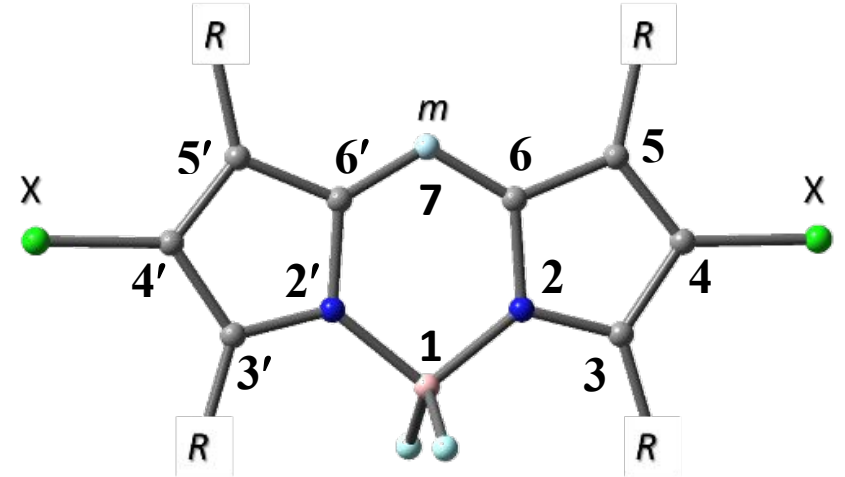

Figure 1. General sketch of XR $m$ BDPs and numbering of their nuclei where $m \mathrm{BDP}$ is a core of such compounds; $m=a, c ; \mathrm{X}$ $=\mathrm{H}, \mathrm{Cl}, \mathrm{Br}, \mathrm{I} ; R=\mathrm{H}$, Methyl $(\mathrm{Me})$, Phenyl $(\mathrm{Ph})$ 
In order to understand the anomalous dependence of the fluorescence quantum yield $\gamma$ upon the nature of the halogen atom and the dependence of the shifts in excited-state energies on structural distortions, the bond lengths between atoms of the $m \mathrm{BDP}$, which is the core for all $\mathrm{XR} m \mathrm{BDP}$ compounds, in the ground and fluorescent excited states are obtained using different computational methods and basis sets, as summarized in Table 1, with the atom numbering given in Figure 1. More details are provided in Supplementary Information Tables SI1-SI3. Solvent effects were not taken into account since the experimental ${ }^{14,16}$ and theoretical ${ }^{23}$ spectra show negligible dependence upon the nature of the solvent used.

All ground-state structures of both halogenated and unsubstituted XPhaBDP compounds optimized using both DFT and MP2 with different basis sets have close to $\mathrm{C}_{2 \mathrm{v}}$ symmetry of the core, with bond length deviations from $\mathrm{C}_{2 \mathrm{v}}$ symmetry being less than $0.002 \AA$ (Table 1, Figure 1 , Table SI1). There are no significant differences between the structures optimized at the HSE06 and MP2 levels of theory though MP2 gives slightly longer bond lengths in the dipyrrin rings compared to the DFT structures. The largest structural differences are $\Delta R=0.013-0.017 \AA$ for the $N_{2}-C_{3}$ and $C_{6}-N_{m}$ bonds, whereas $\Delta R=0.009 \AA$ or less for $C_{5}-C_{6}$. Differences in other bond lengths are negligible. These structural differences do not induce any significant spectral shifts (2-6 nm) in the lowest excited states.

The near $\mathrm{C}_{2 \mathrm{v}}$-symmetric dipyrrin core of the unhalogenated HPhaBDP compounds is maintained for their excited states calculated using different DFT exchange-correlation functionals and basis sets, though somewhat larger deviations from the symmetric structure is observed compared to the ground-state structures. The calculated transition wavelengths to the lowest 
photoactive excited states are lower than the experimental observations by more than $60 \mathrm{~nm}$, and even as large as $100 \mathrm{~nm},{ }^{23}$ see SI. It has previously been reported that $\mathrm{CC} 2$ provides more accurate excitation energies of low-lying excited states, but this approach slightly underestimates these excitation energies in comparison with both experiment and XMCQDPT2 calculations. ${ }^{15}$ In comparison with RI-CC2 (SI), RI-ADC(2)\&dTZVPP significantly improves the wavelengths of the transitions compared to the experiment, but the differences are still large $(40-50 \mathrm{~nm})$ for all compounds (Table 1). The ADC2 method is more time-consuming and memory costly than TDDFT, but provides higher accuracy.

Table 1. Bond lengths in $\AA$ of the $a \mathrm{BDP}$ core conformers optimized at HSE0\&6-311G(d) in the ground and lowest photoactive excited $\left[{ }^{*}\right.$ or ${ }^{\omega}$ at $\left.\omega \mathrm{B} 97 \mathrm{XD} \& 6-311 \mathrm{G}(\mathrm{d})\right]$ states, respectively. The singlet states calculated at ${ }^{1} \mathrm{HSE06} \& 6-311 \mathrm{G}(\mathrm{d})$ and ${ }^{2} \mathrm{ADC}(2) \& \mathrm{dTZVPP}$.

\begin{tabular}{|c|c|c|c|c|c|c|c|c|c|}
\hline bonds groups & $\mathrm{IPh} a 01 *$ & $\operatorname{IPh} a 01^{\omega}$ & $\mathrm{IPh} a 01$ & $\mathrm{BrPh} a 02 *$ & $\mathrm{BrPh} a 02$ & $\mathrm{ClPh} a 03 *$ & ClPh $a 03$ & HPh $a 04 *$ & $\mathrm{HPh} a 04$ \\
\hline $\mathrm{B}_{1}-\mathrm{N}_{2}$ & 1.579 & 1.570 & 1.582 & 1.584 & 1.582 & 1.585 & 1.581 & 1.569 & 1.575 \\
\hline $\mathrm{B}_{1}-\mathrm{N}_{2^{\prime}}$ & 1.556 & 1.571 & 1.582 & 1.555 & 1.582 & 1.554 & 1.581 & 1.569 & 1.575 \\
\hline $\mathrm{N}_{2}-\mathrm{C}_{3}$ & 1.362 & 1.361 & 1.365 & 1.366 & 1.366 & 1.367 & 1.366 & 1.392 & 1.372 \\
\hline $\mathrm{N}_{2^{\prime}}-\mathrm{C}_{3^{\prime}}$ & 1.379 & 1.385 & 1.365 & 1.377 & 1.366 & 1.374 & 1.366 & 1.392 & 1.372 \\
\hline $\mathrm{N}_{2}-\mathrm{C}_{6}$ & 1.391 & 1.383 & 1.380 & 1.390 & 1.381 & 1.389 & 1.381 & 1.377 & 1.383 \\
\hline $\mathrm{N}_{2^{\prime}}-\mathrm{C}_{6^{\prime}}$ & 1.364 & 1.360 & 1.380 & 1.367 & 1.381 & 1.370 & 1.381 & 1.377 & 1.383 \\
\hline $\mathrm{C}_{3}-\mathrm{C}_{4}$ & 1.394 & 1.427 & 1.429 & 1.392 & 1.428 & 1.391 & 1.427 & 1.409 & 1.421 \\
\hline $\mathrm{C}_{3^{\prime}}-\mathrm{C}_{4^{\prime}}$ & 1.421 & 1.429 & 1.429 & 1.420 & 1.428 & 1.419 & 1.428 & 1.409 & 1.421 \\
\hline $\mathrm{C}_{4}-\mathrm{C}_{5}$ & 1.466 & 1.391 & 1.391 & 1.468 & 1.389 & 1.465 & 1.388 & 1.396 & 1.386 \\
\hline $\mathrm{C}_{4^{\prime}}-\mathrm{C}_{5^{\prime}}$ & 1.393 & 1.390 & 1.391 & 1.390 & 1.389 & 1.390 & 1.388 & 1.396 & 1.386 \\
\hline $\mathrm{C}_{5}-\mathrm{C}_{6}$ & 1.387 & 1.439 & 1.442 & 1.389 & 1.442 & 1.392 & 1.441 & 1.459 & 1.442 \\
\hline $\mathrm{C}_{5^{\prime}}-\mathrm{C}_{6^{\prime}}$ & 1.446 & 1.438 & 1.442 & 1.447 & 1.442 & 1.447 & 1.441 & 1.459 & 1.442 \\
\hline $\mathrm{C}_{6}-\mathrm{N}_{m}$ & 1.364 & 1.328 & 1.330 & 1.365 & 1.330 & 1.364 & 1.330 & 1.332 & 1.330 \\
\hline $\mathrm{C}_{6^{\prime}}-\mathrm{N}_{m}$ & 1.306 & 1.329 & 1.330 & 1.305 & 1.330 & 1.305 & 1.330 & 1.332 & 1.330 \\
\hline $\mathrm{C}_{4}-\mathrm{X}$ & 2.051 & 2.088 & 2.097 & 1.847 & 1.876 & 1.670 & 1.718 & 1.09 & 1.090 \\
\hline $\mathrm{C}_{4^{\prime}}-\mathrm{X}$ & 2.093 & 2.089 & 2.097 & 1.878 & 1.876 & 1.722 & 1.718 & 1.09 & 1.090 \\
\hline Max dist. & 0.073 & 0.002 & 0.000 & 0.078 & 0.000 & 0.075 & 0.001 & 0.000 & 0.000 \\
\hline $\begin{array}{l}{ }^{1} \mathrm{~S}_{1}, \mathrm{~nm} \\
{ }^{2} \mathrm{~S}_{1}, \mathrm{~nm}\end{array}$ & 676 & 548 & $\begin{array}{l}550 \\
601\end{array}$ & 663 & $\begin{array}{l}546 \\
601\end{array}$ & 660 & $\begin{array}{l}548 \\
599\end{array}$ & 618 & $\begin{array}{l}561 \\
580\end{array}$ \\
\hline
\end{tabular}




\begin{tabular}{|l|ll|ll|ll|l|l|} 
Exp. $^{16}$ & 674 & 647 & 674 & 643 & 674 & 644 & 675 & 646 \\
\hline
\end{tabular}

Structural relaxation of the excited states is very sensitive to the nature of the particular state. Changes in the structural parameters such as bond lengths of the rings, at which the excitations are localized, lead to spectral shifts. Two distinct natures of the lowest excited state of XPhaBDPs are predicted by the different TD-DFT functionals, differing in their structural symmetry of the dipyrrin core as well as in their electronic properties. The excited states that maintain the near $\mathrm{C}_{2 \mathrm{v}}$ symmetry, IPh $a$ BDP optimized with $\omega$ B97XD\&6-311G(d) for instance, exhibit large blue shifts of the fluorescence wavelengths of up to about $\lambda=100 \mathrm{~nm}$ with large oscillator strengths $(f>0.3)$, compared to the emission spectra of the asymmetric structures $(f=0.04-0.07)$ (Tables 1, SI2 and SI3). Symmetric structures were obtained for the lowest excited states of all the compounds using CAM-B3LYP, wB97XD and M062X. For HSE06, PBE0 and B3LYP, the halogenated molecules have an asymmetric $\mathrm{C}_{1}$ structure in the lowest photoactive singlet states, having the correct wavelengths for the transitions, but with a significantly smaller $f$ (Table 1). The presence of heavy elements essentially distort the symmetry between the 'left' (denoted with the prime symbol in Figure 1) and 'right' parts of XPhaBDPs, leading to a difference $(\Delta)$ of $0.06 \AA-0.08 \AA$ for bondlengths from meso-aza position to the $4,4^{\prime}$-sites of halogenation and a $\Delta$ of $0.03 \AA-0.05 \AA$ for halogens at opposite sites (Table 1). In contrast, the unsubstituted HPhaBDP optimized in the ground and excited states conserves the symmetry in all cases. Heavy halide atoms thus induce notable symmetry distortions in the excited states.

Electronic structure of boron-dipyrromethene derivatives. Changes in the electron density caused by excitation into the first excited singlet state $S_{1}$ of all symmetric compounds is confined to the dipyrrin rings as can be seen from the EDDs $\left(S_{1}-S_{0}\right)$, where the ED of the ground state is 
subtracted from that of the excited state (Figure 2). The transition thus induces a local EDR with negligible charge transfer $(\mathrm{CT})$.

The heavier halogen, the more strongly it contributes to the ED of the excited state. In particular, at the DFT level, iodine donates notably to the excited ED whereas chlorine and bromine hardly contribute to the excited-state ED. The lowest photoactive state of the different compounds has large oscillator strengths $(f=0.5$ for $\mathrm{IPh} a \mathrm{BDP}$ and $f=0.7$ for the remaining molecules) allowing for effective excitation in the longest wavelength band. Minor changes in different bond lengths aimed at improving the calculated wavelengths do not visually change the corresponding EDD, and are therefore not presented here.

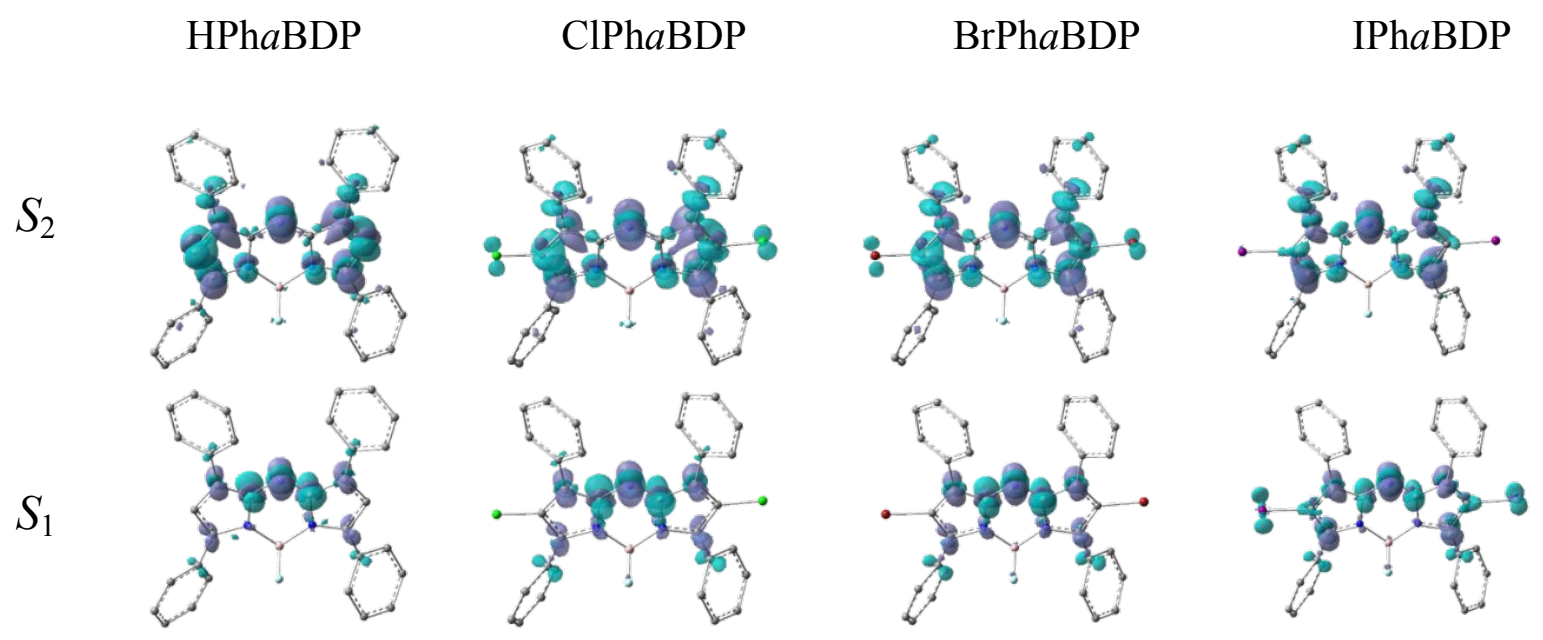

Figure 2. $S_{1}$ and $S_{2}$ excitation EDDs at HSE06\&6-311G(d) spectral calculation of the MP2\&6$311 \mathrm{G}(\mathrm{d})$ optimized XPhaBDPs. Positive EDR direction pointed by the dark lilac color for absorption while the light area means ED loosing.

At the HSE06 level of theory, the second absorptive $S_{2}$ state of all the halides have absorption wavelengths of $517-523 \mathrm{~nm}$, with a somewhat smaller oscillator strength $(f<0.3)$ than for $S_{1}$. For 
$\mathrm{HPh} a \mathrm{BDP}$, the approach gives $476 \mathrm{~nm}$ with lower photoactivity $(f<0.02)$. From Figure 2 it is clear that the EDDs of the $S_{1}$ and $S_{2}$ states are very similar, the main difference being that in the $S_{2}$ state the phenyl rings also act as donors of ED. Comparing the $\mathrm{S}_{1}$ and $\mathrm{S}_{2}$ states, one can see larger EDRs at the ends of the pyrrole rings for the $S_{2}$ state compared to the $S_{1}$ state, also involving CT from peripheral substituents to the dipyrrin core.

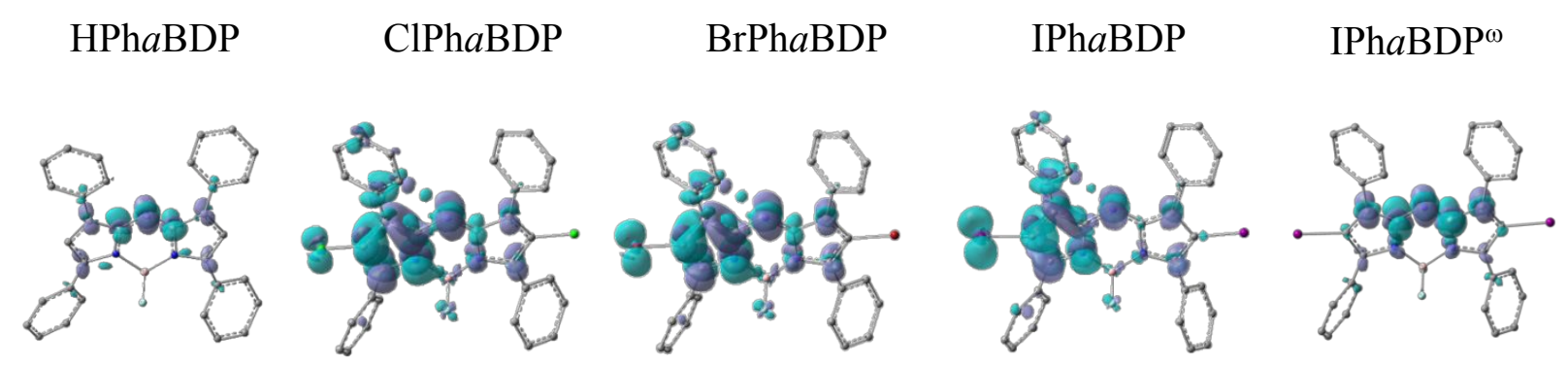

Figure 3. EDD between exited $S_{1}$ and $S_{0}$ of asymmetric HPh $a \mathrm{BDP}, \mathrm{ClPh} a \mathrm{BDP}, \mathrm{BrPh} a \mathrm{BDP}$ and IPh $a \mathrm{BDP}$ (from left to write), optimized at HSE06\&6-311G(d) level of theory and symmetric IPh $a$ BDP relaxed using $\omega \mathrm{B} 97 \mathrm{XD} \& 6-311 \mathrm{G}(\mathrm{d})$ approach where ED redistributes from dark to light lilac color for dissipative transition.

Since the optimized geometries of the electronically excited states are very sensitive to the choice of DFT functional, a small group of computational protocols most suited for fluorescence spectra calculations were selected (Tables SI4-SI8) after extensive tests using six different exchange-correlation functionals and different basis sets (Table SI2). The B3LYP, HSE06 and PBE0 spectral shifts coincide well with experimental data, in contrast to CAM-B3LYP, $\omega \mathrm{B} 97 \mathrm{XD}$ and M062X which gave significant blue shifts of about $100 \mathrm{~nm}$ in the wavelengths of fluorescence compared to experiment. ${ }^{14,16}$ Despite PBE0 producing asymmetric optimized structures, the functional underestimates emission wavelengths by more than $30 \mathrm{~nm}$ compared to B3LYP and HSE06. In contrast to the symmetric compounds, the asymmetric ones, in 
particularly when optimized with HSE06\&6-311G(d), lead to localization of EDR on one side of the molecules (Figure 3) upon excitation. The EDD for the emission processes are calculated as for absorption, but since emission is a reverse dissipative process, the sign of the changes in the ED is reversed.

$$
\begin{aligned}
& X=1 \\
& S_{2} \underline{558(0.70)} \quad S_{2} \text { 562(0.67), 566(0.64) } \\
& S_{1} \underline{676(0.04)} \quad \underline{673} T_{3} \\
& S_{1} \stackrel{663(0.04), 655(0.07)}{664,660} T_{3} \\
& 880 T_{2} \quad \underline{879,871} T_{2}
\end{aligned}
$$

Figure 4. A schematic representation of the lowest excited states of the XPhaBDP fluorescent structures optimized at HSE06\&6-311G(d), explaining the higher fluorescence efficiency $\gamma$ in IPhaBDP than in BrPhaBDP (see text). The presented values of energies in $\mathrm{nm}$ and oscillator strengths $f$ in parenthesis.

Calculations with the different functionals and basis sets described in the Computational Methods section identify a triplet state (usually $T_{3}$ ) very close in energy and below the $S_{1}$ state. This can give a rise to a significant SOC of these states caused by the heavy halogens and thus a competition between the rates of fluorescence and ISC. The fluorescence efficiency $\gamma$ should therefore decrease with increasing atomic number of the halogen atom if the electronic structures otherwise remain the same. As noted previously, this is however not observed experimentally.

To explain and illustrate the origins of the breakdown of this expected trend, the most suitable fluorescent molecular structures were optimized and their several lowest excited states were calculated using, the most acceptable for this case, TD-HSE06\&6-311G(d) computational level, which performs the best spectral-luminescence properties compared to experiment (Figures 4 and SI1). The calculations provide an inversion of the $S_{1}$ and $T_{3}$ states for IPhaBDP, whereas the energy gap between the $S_{1}$ and $T_{2}$ states is large enough to prevent a significant ISC. The fact, that $T_{3}$ is 
higher in energy than the $S_{1}$ in IPhaBDP and $S_{1}-T_{3}$ energy gap is very narrow in BrPhaBDP, could explain the experimentally observed much more effective fluorescence of IPhaBDP $(\gamma=0.33)$ compared BrPhaBDP $(\gamma=0.03)$. The lowest triplet state $T_{1}$ was not considered at all because the energy gap to $S_{1}$ in all cases are very large that leads to a negligible ISC probability between these states for all molecules. Internal conversions are not discussed due to huge energy gaps between the $S_{1}$ and ground state and thus not in competition with fluorescence and ISC processes.

Spectral-luminescence properties. The fluorescence efficiency or quantum yield $\gamma$ is defined as the emission rate divided by the total rate of dissipative transitions from the same exited state and is expressed as $\gamma=k_{r} /\left(k_{r}+k_{\text {isc }}\right)$ if internal conversions are neglected, as is usual for organic compounds due to the large energy separation of the fluorescent and ground electronic states. The rate constant of fluorescence is calculated as $k_{r}=\sqrt{2} E_{S_{1}}^{2} f$ in s ${ }^{-1}$, where the oscillator strength $f$ is dimensionless and $E_{S 1}$ is the energy separation of the ground and excited states in $\mathrm{cm}^{-1}$.

In the adiabatic approximation, the movement of the atoms during fast EDRs is neglected. In absorption and fluorescence processes, transition rates of about $10^{-8} \mathrm{~s}^{-1}$ are so fast that the nuclear framework in general can be assumed to not change during the electronic excitation/de-excitation processes, being fixed at the zero-point vibrationally averaged structure of the initial state, that is, the ground state for absorption processes or the excited state for fluorescence processes. In the case when the fluorescence $\gamma$ is less than $100 \%$, the ISC rate is of the same order of magnitude or faster than the fluorescence rates, making the adiabatic approximation a good one. In this case, the geometry optimized in its fluorescent state is used to calculate the ISC rates from this electronic state to lower unrelaxed triplet states. 
Energy gaps and magnitudes of SOC-ME between the singlet and triplet states are two factors which determine the ISC rates, and thus determine quenching of the fluorescence. There are different approaches to calculate the ISC rate $k_{i s c}$, from the simplest used for light elements where the spin and orbit parts can be separated ${ }^{11,24,52-55}$ through several more accurate approaches ${ }^{56-58}$. The ISC rates are expressed as $k_{i s c}=\frac{2 \pi}{\hbar}\left\langle S\left|H_{s o}\right| T\right\rangle^{2} \rho_{F C}$, where the Frank-Condon weighted density of states can be evaluated as $\rho_{F C}=\frac{1}{\sqrt{4 \pi \lambda_{M} k_{B} T}} \exp \left[-\frac{\left(\Delta E+\lambda_{M}\right)^{2}}{4 \lambda_{M} k_{B} T}\right]$ for medium-sized molecules according to the Marcus-Levich-Jortner theory ${ }^{12,24,55,59} \cdot \rho_{F C}$ depends on standard thermodynamical parameters, the energy gap $\Delta E$ and the Marcus reorganization energy $\lambda_{M}$, for which recommended values are in the range $\sim 0.1-0.2 \mathrm{eV}^{55}$. The largest recommended Marcus reorganization energy was used in this work to reach the best agreement with experimental observations.

Excited-state structures and spectral properties were calculated using HSE06\&6-311G(d). However, SOC-ME's are not available at the DFT level in G09. Both ADF and Dalton can calculate SOC-ME at the DFT level, but the functional and basis sets identified as the best for the spectral properties in the previous section are not available in these codes. Supplementary Information contains details and comparison of SOC-ME calculations using both HSE06 and the widespread B3LYP functional (Table SI9), in particular, implemented in G09, TM7.2, Dalton and ADF to ensure that the MolSOC results are adequate to the values obtained in all the packages.

The narrow energy gaps between fluorescent $S_{1}$ and $T_{3}$ states in XPhaBDPs satisfy requirements for an effective ISC (Table 2, Figure 4). Heavy elements increase the SOC and, subsequently, the ISC rates. Iodine should lead to almost total quenching of the fluorescence, but the energy of the $S_{1}$ state is lower-lying than that of the $T_{3}$ state. Various possibilities of mutual 
positions and energy gaps between these states in IPh $a \mathrm{BDP}$ and $\mathrm{BrPh} a \mathrm{BDP}$ (SI1, SI2 and the most acceptable results in SI4) using numerous settings of several DFT functionals with various basis sets (see Computational Details) were tested and only the one that best reflects the experimental data were described in the text. Moreover, there is an uncertainty between the acceptable error about $0.1 \mathrm{eV}$ and even larger [23] in theoretical energies of the excited states and the required narrow energy gaps between the triplet and the singlet state, particularly this is less than $0.02 \mathrm{eV}$ for $\mathrm{BrPh} a \mathrm{BDP}$, to reach an acceptable fluorescence quantum yield.

Table 2. Wavelengths (nm) and root mean squares ( $\left.r m s=\sqrt{\sum_{i=x, y, z}\left\langle S\left|H_{s o}\right| T\right\rangle_{i}^{2}}\right)$ SOC-ME $\left(\mathrm{cm}^{-1}\right)$ of transitions from $S_{1}$ to triplets with corresponding dimensionless fluorescence efficiency $\gamma$ (in curly brackets) for XPhaBDP calculated for the excited states with oscillator strength $f$ (a.u.) for singlets are presented in parentheses. The rates of fluorescence $v s$ ISC $\left(\mathrm{s}^{-1}\right)$ are under each $\gamma$.

\begin{tabular}{|l|l|l|l|l|}
\hline states & IPh $a$ BDP & BrPh $a$ BDP & ClPh $a$ BDP & HPh $a$ BDP \\
\hline $\mathrm{T}_{3}$ & $\begin{array}{l}673\{9.09 ; \gamma=0.00\} \\
6.19 \times 10^{6} v s 6.36 \times 10^{10}\end{array}$ & & & \\
$\mathrm{~S}_{1}$ & $676(f=0.04)$ & $663(f=0.04)$ & $655(f=0.07)$ & $614(f=0.65)$ \\
$\mathrm{T}_{3}$ & & $\begin{array}{l}664\{3.46 ; \gamma=0.01\} \\
6.43 \times 10^{6} v s 9.41 \times 10^{8}\end{array}$ & $\begin{array}{l}660\{1.38 ; \gamma=0.09\} \\
1.15 \times 10^{7} v s 1.19 \times 10^{8}\end{array}$ & $\begin{array}{l}620\{0.37 ; \gamma=0.93\} \\
1.22 \times 10^{8} v s 7.86 \times 10^{6} \\
\mathrm{~T}_{2}\end{array}$ \\
$\begin{array}{l}880\{29.96 ; \gamma=1.00\} \\
6.19 \times 10^{6} v s 3.26 \times 10^{2}\end{array}$ & $879\{4.96\}$ & $871\{0.68\}$ & $633\{0.23\}$ \\
\end{tabular}

At the same time, the energy gap between $S_{1}$ and the next lower-energy $\mathrm{T}_{2}$ state is large enough to prevent ISC and thus reach maximum fluorescence even if the value of the SOC-ME is significant. On the other hand, the $S_{1}$ and $T_{3}$ states are almost degenerate, allowing one to consider the possibility of state flipping under specific environmental conditions. Based on the available experimental data and the results of electronic structure calculations, it is reasonable to assume 
that IPhaBDP can be in a mixture of fully effective and quenched fluorescent states, so that statistically a fluorescence efficiency of one third can be assumed at a macroscopic level. Alternatively, if the $T_{2}$ energy is assumed to be underestimated, then this state should be at a wavelength of about $\lambda=770 \mathrm{~nm}$ (frequency $v=13000 \mathrm{~cm}^{-1}$ ), that is $110 \mathrm{~nm}$ shorter than the calculated value, with the same $S_{1} \rightarrow T_{2}$ SOC-ME's to provide an agreement with experimental fluorescence quantum yield. ${ }^{16}$ The fluorescence efficiency in BrPhaBDP is observed experimentally as $\gamma=0.03^{14,16,17}$ which is close to the MolSOC calculation of ME $=0.01$ (Table 2) and one could assume that the energy of the $T_{3}$ state is always lower than $S_{1}$ for this molecule (Figure 4). The energy gap between the $T_{3}$ and $S_{1}$ states for $\mathrm{ClPh} a \mathrm{BDP}$ is wider and coincide well with the experimental and calculated $\gamma$ of about 0.3 and 0.1 , respectively. ${ }^{17}$ The fluorescence efficiency of the halogen-unsubstituted molecule is close to unity, ${ }^{15}$ independently of the ordering of the excited states, although the calculated results are in better agreement with experimental data if $S_{1}$ is lower than $T_{3}$, giving $\gamma=0.88$, but compared to assuming $S_{1}$ to be higher in energy, the difference is small, $\gamma=0.94$.

Table 3. XRmBDP optimized in the lowest photoactive state and its spectral properties calculated using HSE06\&6-311G(d). Dimensionless oscillator strength $f$ of $S_{1}$ and $S_{2}(\mathrm{~nm})$ are presented in parentheses, the rms SOC-ME $\left(\mathrm{cm}^{-1}\right)$ between $S_{1}$ and $T_{2}$ or $T_{3}$ for significant ISC and corresponding partial $\gamma$ (a.u.) are presented in curly brackets.

\begin{tabular}{|l|l|l|l|l|}
\hline OptS $_{1} \&$ TD & \multicolumn{1}{|c|}{ IR $m$ BDP } & \multicolumn{1}{c|}{ BrR $m$ BDP } & \multicolumn{1}{c|}{ ClR $m$ BDP } & \multicolumn{1}{c|}{ HR $m$ BDP } \\
\hline XH $a$ BDP & 624 & 596 & 583 & 524 \\
& $646(0.11)$ & $635(0.08)$ & $631(0.07)$ & $571(0.02)$ \\
& 780 & 805 & 813 & 787 \\
\hline XMe $a$ BDP & 582 & 568 & 562 & 514 \\
& $607(0.11)$ & $605(0.08)$ & $605(0.06)$ & $563(0.02)$ \\
& 757 & 774 & 786 & 747 \\
\hline XPh $a$ BDP & $558(0.70)$ & $562(0.67)$ & $566(0.64)$ & $498(0.03)$ \\
& $673\{9.09 ; 0.00\}$ & $663(0.04)$ & $655(0.07)$ & $614(0.65)$ \\
& $676(0.04)$ & $664\{3.46 ; 0.01\}$ & $660\{1.19 ; 0.09\}$ & $620\{0.37 ; 0.93\}$ \\
\hline
\end{tabular}




\begin{tabular}{|l|l|l|l|l|}
\hline & $880\{29.96 ; 1.00\}$ & 879 & 871 & 633 \\
\hline XPhcBDP & $514(0.60)$ & $511(0.68)$ & $513(0.70)$ & 513 \\
& $535\{15.32 ; 0.072\}$ & $519\{5.25 ; 0.15\}$ & $517\{0.45 ; 0.94\}$ & 515 \\
& $558\{18.44 ; 0.55\}$ & $545\{2.81 ; 0.94\}$ & $543\{0.94 ; 0.99\}$ & $531(0.54)$ \\
\hline
\end{tabular}

Other XRmBDP molecular structures were also optimized in the lowest photoactive singlet states and their emission spectra were calculated using HSE06\&6-311G(d). These compounds were compared with $\mathrm{XPh} a \mathrm{BDPs}$ to inquire whether the increase in fluorescence for IPh $a \mathrm{BDP}$ is unique for this class of molecules or it applies to all halogenated XRmBDPs (Table 3).

The most prominent influence of 3,3',5,5'-tetra substitutions and nitrogen in the meso-position are on the shifts in emission wavelengths. The $T_{3}$ states in the tetra-methyl substituted and 3,3',5,5' unsubstituted aza-compounds have higher energies than the $S_{1}$ states, and thus give rise to an effective fluorescence with slightly shorter wavelengths than XPh $a$ BDPs. Fluorescence quenching cannot be expected in other XRaBDPs $(\mathrm{R}=\mathrm{H}, \mathrm{Me})$ because the energy of the $T_{3}$ state is higherlying than the $S_{1}$ state for all cases and the gap is significantly wider than for XPhaBDP, but at the same time the $T_{2}$ energy is low enough to prevent any notable ISC to this state.

To the best of our knowledge, there is no experimental data for the halogenated $\mathrm{XPh} c \mathrm{BDP}$ compounds with carbon in the meso-position. The electronic structure calculations presented in this work predict low fluorescence efficiency because the $T_{3}$ energy is slightly lower-lying than the $S_{1}$ one, facilitating large SOCs. Quantum yields are normally decreasing with increasing weight of the substituents due to stronger SOC and because the energy gaps between these states is larger than in the XPhaBDP cases, preventing an inversion between the $T_{3}$ and $S_{1}$ states. In contrast with the aza-compounds, the $S_{1}$ energy is the highest among these three states for XPhcBDPs (Table 3 ), but the energy gap between the $S_{1}$ and $T_{2}$ states is not so large as to prevent SOC of these states. Both the $T_{2}$ and $T_{3}$ states effectively quench fluorescence, but this is counteracted by the strong $f$, 
thus still supporting non-negligible fluorescence efficiencies. The balance between the rates of these transitions define the dependency on halogen substituents, which in turn is due to the relative ordering of the excited singlet and triplet states in these molecules.

Interaction between XPh $a$ BDP and $\mathbf{O}_{2}$. The asymmetric molecules undergo significant EDR upon excitation into the lowest photoactive excited state, leading to charge separation in the excited state (Figures 3 and 5). The ESP map is used to identify the most probable sites for ion (either anion or cation) attack in the excited state as well as to simulate fluorescence quenching through energy transfer due to triplet sensitization in complexes with $\mathrm{O}_{2}$.

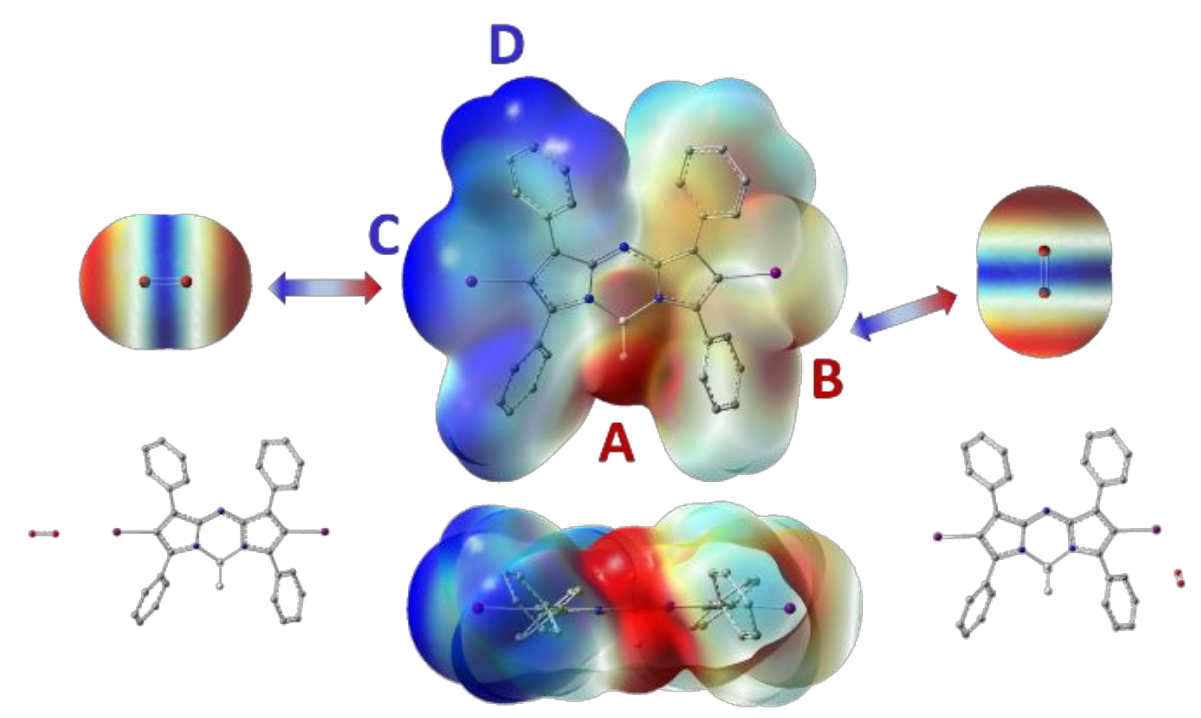

Figure 5. Mapping from $-25.1 \mathrm{kcal} / \mathrm{mol}$ (red) to $+12.6 \mathrm{kcal} / \mathrm{mol}$ (blue) ESP on the isodensity of $0.004 \mathrm{e}^{-} / \mathrm{bohr}^{3}$ to couple with $\mathrm{O}_{2}$ on long distances.

The best configuration of the complex that allows for the most effective $T \rightarrow T$ energy transfer from the donor to the oxygen acceptor is when the ED on both molecules are as close as possible to facilitate overlap of their EDs, and with electropositive and electronegative regions (or vice 
versa) of the two molecules facing each other. Such interactions occur in collision complexes that should be studied in dynamics simulations, but the best configurations for such processes can be predicted based on an ESP analysis. The EDR following the dissipative photo-transition from the lowest excited state induces four notably charged areas with negative (A and B) and positive (C and D) regions where $\mathrm{O}_{2}$ can attack the compounds (Figure 5).

The ESP maps are qualitatively the same for all the halides, but the values of the extreme points are slightly different. Point A provides the absolute ESP minima $-31.4 \mathrm{kcal} / \mathrm{mol},-28.2 \mathrm{kcal} / \mathrm{mol}$ and $-27.6 \mathrm{kcal} / \mathrm{mol}$ for $\mathrm{I}, \mathrm{Br}$ and $\mathrm{Cl}$ substituents on the $\mathrm{ED}$ isosurface of $0.004 \mathrm{e}^{-} / \mathrm{bohr}^{3}$, respectively. The minima are localized near the fluorine atoms but very close to the phenyl rings, which impose steric hindrance to the site and thus preventing collision complexes with $\mathrm{O}_{2}$ at this site. The value for $\mathrm{D}$ is very similar for all halides on a near equipotential surface, ranging from $+19.5 \mathrm{kcal} / \mathrm{mol}$ to $+20.7 \mathrm{kcal} / \mathrm{mol}$. The positive potential delocalized on a large area over the phenyl rings can enable interactions and even collisions with $\mathrm{O}_{2}$, but these fragments can take no part in the dissipative transitions from the lowest photoactive state and, therefore, do play no role in following $T-T$ processes. This is the smallest 'left' side of the asymmetric structures, whereas the excitation is largely localized on the 'right' (and "largest") part (Table 1 and Figures 1, 3).

The positive $\mathrm{C}$ and negative $\mathrm{B}$ sites are the most promising for interactions between the compounds studied here and $\mathrm{O}_{2}$, with the possibility of energy transfer to produce a triplet complex. The most negative point, $-27.6 \mathrm{kcal} / \mathrm{mol}$, is induced with participation of the iodine substituents, whereas bromine and chlorine provide weaker minima of $-20.7 \mathrm{kcal} / \mathrm{mol}$ and -19.5 $\mathrm{kcal} / \mathrm{mol}$, respectively. The maximum positive value of $+28.9 \mathrm{kcal} / \mathrm{mol}$ on the opposite end is also induced by iodine, whereas bromine and chlorine give $+18.8 \mathrm{kcal} / \mathrm{mol}$ and $+12.6 \mathrm{kcal} / \mathrm{mol}$, 
respectively. IPh $a \mathrm{BDP}$ exhibits the highest reactivity on both ends whereas $\mathrm{BrPh} a \mathrm{BDP}$ and ClPhaBDP have similar interaction strengths with $\mathrm{O}_{2}$ at the site between the halogen and the phenyl segment, whereas ClPhaBDP has the weakest attraction to $\mathrm{O}_{2}$ along the axis through both molecules. Thus, BrPhaBDP should be the most effective triplet photosensitizer taking into account the lower fluorescence efficiency of this compound.

\section{CONCLUSIONS}

The spectral-luminescent properties of dihalogen-tetraphenyl-aza-BDP (XPhaBDP) molecules optimized in their ground and fluorescent electronic states were studied using a wide set of quantum-mechanical methods that includes several TD-DFT methods as well as RI-ADC(2) and RI-CC2 with various basis sets. Theoretical results of the best samples correspond well to available experimental spectral data. The dependencies between the symmetries of the compounds and energies of the lowest excited states were established. It was found that calculations on the symmetric molecules gave too short long bond lengths in the dipyrrin rings, leading to significant spectral blue shifts in comparison with the asymmetric counterparts, which provide perfect agreement with experimental fluorescence spectra. The electron density redistribution in the excited state of the asymmetric compounds largely occurs at one end of the molecule, and has the correct wavelength of the lowest photoactive singlets. The best agreement between the calculated and measured $\mathrm{XPh} a \mathrm{BDP}$ spectral-luminescent properties was achieved with a combination of the HSE06 functional and Pople split-valence diffuse 6-311G*(Cl, Br, I)/3-21G(H)/6-31G* basis set.

The halogen and phenyl groups provide a competition between fluorescence and intersystem crossing due to the small energy gaps between the lowest near-lying photoactive singlet and triplet states as well as significant spin-orbit coupling between the states. The balance between 
probabilities of these dissipative transitions was established to estimate correct quantum yields. Significant luminescent bathochromic shifts of more than $100 \mathrm{~nm}$ and inversion between the fluorescent state and the nearest triplet state to possess a few higher energy in IPh $a \mathrm{BDP}$ are induced by aza substitution into the meso-position, explaining the abnormal "gravity" effect (fluorescence quantum yield should be decreasing with increasing atomic number of the halogen atom), namely that the energy gap between the fluorescence state and the next much lower-lying triplet state is large enough to prevent any significant ISC, and thus that a higher fluorescence efficiency is observed for the heavier halogen-substituted $\operatorname{IPh} a \operatorname{BDP}(\gamma=0.33)$ than in $\operatorname{BrPh} a \operatorname{BDP}(\gamma=0.03)$.

High rate of fluorescence quenching by ISCs and probabilities of collisions between the compounds and oxygen molecules in a solvent should provide effective triplet sensitization. The most preferable sites for such interactions were predicted using ESP mapping at the extreme positive and negative charge points. The two most preferable sites were found to be near the halogen substituents for interaction between the compounds and oxygen molecules in their collision complexes and thus to provide an effective triplet-triplet energy transfer.

\section{ASSOCIATED CONTENT}

Supporting Information contains geometric parameters of the key structures and photophysical properties calculated using various quantum-mechanical methods.

\section{ACKNOWLEDGMENTS}

The research was supported by Basic Science Research Program through the National Research Foundation of Korea (NRF) funded by the Ministry of Education (Grant No. 2019R1I1A1A01061339). All calculations were implemented in Norway with the support of the 
Norwegian Supercomputer Program (Grant No. NN4654K). The work in Tromsø was supported

by the Research Council of Norway through a Centre of Excellence Grant (Grant No. 262695) and Research Grant 250743.

\section{REFERENCES:}

(1) Dye Lasers, 3rd enl. and rev. ed.; Schäfer, F. P., Drexhage, K. H., Eds.; Topics in applied physics; Springer-Verlag: Berlin; New York, 1990.

(2) Pavlopoulos, T. G.; Boyer, J. H.; Shah, M.; Thangaraj, K.; Soong, M.-L. Laser Action from 2,6,8Position Trisubstituted 1,3,5,7-Tetramethylpyrromethene-BF_2 Complexes: Part 1. Appl. Opt. 1990, 29, 3885.

(3) Cerdán, L.; Costela, A.; García-Moreno, I.; García, O.; Sastre, R. Laser Emission from Mirrorless Waveguides Based on Photosensitized Polymers Incorporating POSS. Opt. Express 2010, 18, 10247.

(4) Kuznetsova, R. T.; Aksenova, Y. V.; Solodova, T. A.; Kopylova, T. N.; Tel'minov, E. N.; Mayer, G. V.; Berezin, M. B.; Antina, E. V.; Burkova, S. L.; Semeikin, A. S. Lasing Characteristics of Difluoroborates of 2,2'-Dipyrromethene Derivatives in Solid Matrices. Quantum Electron. 2014, 44, 206-212.

(5) Loudet, A.; Burgess, K. BODIPY Dyes and Their Derivatives: Syntheses and Spectroscopic Properties. Chem. Rev. 2007, 107, 4891-4932.

(6) Ermolina, E. G.; Kuznetsova, R. T.; Aksenova, Yu. V.; Gadirov, R. M.; Kopylova, T. N.; Antina, E. V.; Berezin, M. B.; Semeikin, A. S. Novel Quenchometric Oxygen Sensing Material Based on Diiodine-Substituted Boron Dipyrromethene Dye. Sens. Actuators B Chem. 2014, 197, 206-210.

(7) Kuznetsova, R. T.; Aksenova, Iu. V.; Bashkirtsev, D. E.; Shulev, A. S.; Antina, E. V.; Berezin, M. B.; Bumagina, N. A. Determination of the Quantum Yield of Singlet Oxygen Sensitized by Halogenated Boron Difluoride Dipyrromethenes. High Energy Chem. 2017, 51, 175-181.

(8) Sánchez-Arroyo, A. J.; Palao, E.; Agarrabeitia, A. R.; Ortiz, M. J.; García-Fresnadillo, D. Towards Improved Halogenated BODIPY Photosensitizers: Clues on Structural Designs and Heavy Atom Substitution Patterns. Phys. Chem. Chem. Phys. 2017, 19, 69-72.

(9) Epelde-Elezcano, N.; Martínez-Martínez, V.; Peña-Cabrera, E.; Gómez-Durán, C. F. A.; Arbeloa, I. L.; Lacombe, S. Modulation of Singlet Oxygen Generation in Halogenated BODIPY Dyes by Substitution at Their Meso Position: Towards a Solvent-Independent Standard in the Vis Region. RSC Adv. 2016, 6, 41991-41998.

(10) Sinel'nikov, A. N.; Artyukhov, V. Ya.; Aksenova, Yu. V.; Kuznetsova, R. T. Photophysics of the Analogs of Pyrromethene PM 567. Opt. Spectrosc. 2012, 113, 291-297.

(11) Sinel'nikov, A. N.; Aksenova, Iu. V.; Kuznetsova, R. T.; Artyukhov, V. Ya.; Bashkirtsev, D. E.; Pozdnyakov, I. P.; Berezin, M. B. Photophysics of Boron Difluoride Chelates with Dihalogenated Tetraphenyl-Ms-Azadipyrromethenes. High Energy Chem. 2016, 50, 266-273.

(12) De Simone, B. C.; Mazzone, G.; Pirillo, J.; Russo, N.; Sicilia, E. Halogen Atom Effect on the Photophysical Properties of Substituted Aza-BODIPY Derivatives. Phys. Chem. Chem. Phys. 2017, 19, 2530-2536. 
Nikonova, A. Yu.; Kuznetsova, R. T.; Aksenova, Iu. V.; Tel'minov, E. N.; Mayer, G. V.; Dudina, N. A.; Nuraneeva, E. N.; Antina, E. V. Optical Properties of Zinc(II) and Boron(III) Dipyrrinates with Different Structures. Opt. Spectrosc. 2016, 120, 395-402.

Kuznetsova, R. T.; Aksenova, I. V.; Bashkirtsev, D. E.; Prokopenko, A. A.; Pomogaev, V. A.; Antina, E. V.; Berezin, M. B.; Bumagina, N. A. Photonics of Coordination Complexes of Dipyrrins with P- and d-Block Elements for Application in Optical Devices. J. Photochem. Photobiol. Chem. 2018, 354, 147-154.

Valiev, R. R.; Sinelnikov, A. N.; Aksenova, Y. V.; Kuznetsova, R. T.; Berezin, M. B.; Semeikin, A. S.; Cherepanov, V. N. The Computational and Experimental Investigations of Photophysical and Spectroscopic Properties of BF2 Dipyrromethene Complexes. Spectrochim. Acta. A. Mol. Biomol. Spectrosc. 2014, 117, 323-329.

(16) Kuznetsova, R. T.; Aksenova, Iu. V.; Prokopenko, A. A.; Pomogaev, V. A.; Antina, E. V.; Berezin, M. B.; Antina, L. A.; Bumagina, N. A. Photonics of Boron(III) and Zinc(II) Dipyrromethenates as Active Media for Modern Optical Devices. J. Mol. Liq. 2019, 278, 5-11. Aksenova, I. V.; Kuznetsova, R. T.; Pozdnyakov, I. P.; Plyusnin, V. F.; Berezin, M. B.; Bumagina, N. A.; Jarnikova, E. S.; Parkhats, M. V.; Dzhagarov, B. M. Spectral-Kinetic Properties and Efficiency of Singlet Oxygen Generation by Some Dipyrromethenes. J. Photochem. Photobiol. Chem. 2017, 344, 206-211. Ullrich, C. Time-Dependent Density-Functional Theory: Concepts and Applications; Oxford graduate texts; Oxford University Press: Oxford ; New York, 2012.

Adamo, C.; Jacquemin, D. The Calculations of Excited-State Properties with Time-Dependent Density Functional Theory. Chem Soc Rev 2013, 42, 845-856.

Adamo, C.; Le Bahers, T.; Savarese, M.; Wilbraham, L.; García, G.; Fukuda, R.; Ehara, M.; Rega, N.; Ciofini, I. Exploring Excited States Using Time Dependent Density Functional Theory and Density-Based Indexes. Coord. Chem. Rev. 2015, 304-305, 166-178.

Ksenofontov, A. A.; Guseva, G. B.; Antina, E. V. Theoretical Studies on the Electronic Structure and Spectroscopic Properties of Transition Metals Bis (Dipyrrinate)s. Mol. Phys. 2016, 114, 28382847.

Le Guennic, B.; Maury, O.; Jacquemin, D. Aza-Boron-Dipyrromethene Dyes: TD-DFT Benchmarks, Spectral Analysis and Design of Original near-IR Structures. Phys Chem Chem Phys 2012, 14, 157-164.

Momeni, M. R.; Brown, A. Why Do TD-DFT Excitation Energies of BODIPY/Aza-BODIPY Families Largely Deviate from Experiment? Answers from Electron Correlated and Multireference Methods. J. Chem. Theory Comput. 2015, 11, 2619-2632.

Schmidt, K.; Brovelli, S.; Coropceanu, V.; Beljonne, D.; Cornil, J.; Bazzini, C.; Caronna, T.; Tubino, R.; Meinardi, F.; Shuai, Z.; et al. Intersystem Crossing Processes in Nonplanar Aromatic Heterocyclic Molecules. J. Phys. Chem. A 2007, 111, 10490-10499. Hohenberg, P.; Kohn, W. Inhomogeneous Electron Gas. Phys. Rev. 1964, 136, B864-B871. Kohn, W.; Sham, L. J. Self-Consistent Equations Including Exchange and Correlation Effects. Phys. Rev. 1965, 140, A1133-A1138.

Koch, W.; Holthausen, M. C. A Chemist's Guide to Density Functional Theory, 2nd ed., 5. reprint.; Wiley-VCH: Weinheim, 2008.

Parr, R. G.; Yang, W. Density-Functional Theory of Atoms and Molecules, 1. iss. as ... paperback.; International series of monographs on chemistry; Oxford Univ. Press [u.a.]: New York, NY, 1994. 
(29) Adamo, C.; Barone, V. Toward Reliable Density Functional Methods without Adjustable Parameters: The PBE0 Model. J. Chem. Phys. 1999, 110, 6158.

(30) Becke, A. D. Density-Functional Exchange-Energy Approximation with Correct Asymptotic Behavior. Phys. Rev. A 1988, 38, 3098-3100.

(31) Lee, C.; Yang, W.; Parr, R. G. Development of the Colle-Salvetti Correlation-Energy Formula into a Functional of the Electron Density. Phys. Rev. B 1988, 37, 785-789.

(32) Zhao, Y.; Truhlar, D. G. The M06 Suite of Density Functionals for Main Group Thermochemistry, Thermochemical Kinetics, Noncovalent Interactions, Excited States, and Transition Elements: Two New Functionals and Systematic Testing of Four M06-Class Functionals and 12 Other Functionals. Theor. Chem. Acc. 2008, 120, 215-241.

(33) Heyd, J.; Scuseria, G. E.; Ernzerhof, M. Erratum: "Hybrid Functionals Based on a Screened Coulomb Potential" [J. Chem. Phys. 118, 8207 (2003)]. J. Chem. Phys. 2006, 124, 219906.

(34) Izmaylov, A. F.; Scuseria, G. E.; Frisch, M. J. Efficient Evaluation of Short-Range Hartree-Fock Exchange in Large Molecules and Periodic Systems. J. Chem. Phys. 2006, 125, 104103.

(35) Chai, J.-D.; Head-Gordon, M. Systematic Optimization of Long-Range Corrected Hybrid Density Functionals. J. Chem. Phys. 2008, 128, 084106.

(36) Chai, J.-D.; Head-Gordon, M. Long-Range Corrected Hybrid Density Functionals with Damped Atom-Atom Dispersion Corrections. Phys. Chem. Chem. Phys. 2008, 10, 6615.

(37) Yanai, T.; Tew, D. P.; Handy, N. C. A New Hybrid Exchange-Correlation Functional Using the Coulomb-Attenuating Method (CAM-B3LYP). Chem. Phys. Lett. 2004, 393, 51-57.

(38) Frisch, M. J.; Trucks, G. W.; Schlegel, H. B.; Scuseria, G. E.; Robb, M. A.; Cheeseman, J. R.; Scalmani, G.; Barone, V.; Mennucci, B.; Petersson, G. A.; et al. Gaussian, 09, Revision D.01, Gaussian Inc., Wallingford CT, 2013.

(39) Eichkorn, K.; Treutler, O.; Öhm, H.; Häser, M.; Ahlrichs, R. Auxiliary Basis Sets to Approximate Coulomb Potentials. Chem. Phys. Lett. 1995, 240, 283-290.

(40) Dunning, T. H. Gaussian Basis Sets for Use in Correlated Molecular Calculations. I. The Atoms Boron through Neon and Hydrogen. J. Chem. Phys. 1989, 90, 1007-1023.

(41) Clark, T.; Chandrasekhar, J.; Spitznagel, G. W.; Schleyer, P. V. R. Efficient Diffuse FunctionAugmented Basis Sets for Anion Calculations. III. The 3-21+G Basis Set for First-Row Elements, Li-F. J. Comput. Chem. 1983, 4, 294-301.

(42) Frisch, M. J.; Head-Gordon, M.; Pople, J. A. A Direct MP2 Gradient Method. Chem. Phys. Lett. 1990, 166, 275-280.

(43) Weigend, F.; Ahlrichs, R. Balanced Basis Sets of Split Valence, Triple Zeta Valence and Quadruple Zeta Valence Quality for H to Rn: Design and Assessment of Accuracy. Phys. Chem. Chem. Phys. 2005, 7, 3297.

(44) Schirmer, J. Beyond the Random-Phase Approximation: A New Approximation Scheme for the Polarization Propagator. Phys. Rev. A 1982, 26, 2395-2416.

(45) Hättig, C. Structure Optimizations for Excited States with Correlated Second-Order Methods: CC2 and ADC(2). In Advances in Quantum Chemistry; Elsevier, 2005; Vol. 50, pp 37-60.

(46) Furche, F.; Ahlrichs, R.; Hättig, C.; Klopper, W.; Sierka, M.; Weigend, F. Turbomole. Wiley Interdiscip. Rev. Comput. Mol. Sci. 2014, 4, 91-100.

(47) te Velde, G.; Bickelhaupt, F. M.; Baerends, E. J.; Fonseca Guerra, C.; van Gisbergen, S. J. A.; Snijders, J. G.; Ziegler, T. Chemistry with ADF. J. Comput. Chem. 2001, 22, 931-967. 
(48) Aidas, K.; Angeli, C.; Bak, K. L.; Bakken, V.; Bast, R.; Boman, L.; Christiansen, O.; Cimiraglia, R.; Coriani, S.; Dahle, P.; et al. The Dalton Quantum Chemistry Program System: The Dalton Program. Wiley Interdiscip. Rev. Comput. Mol. Sci. 2014, 4, 269-284.

(49) Chiodo, S. G.; Russo, N. One-Electron Spin-Orbit Contribution by Effective Nuclear Charges. $J$. Comput. Chem. 2009, 30, 832-839.

(50) Chiodo, S. G.; Russo, N. DFT Spin-Orbit Coupling between Singlet and Triplet Excited States: A Case of Psoralen Compounds. Chem. Phys. Lett. 2010, 490, 90-96.

(51) Chiodo, S. G.; Leopoldini, M. MolSOC: A Spin-Orbit Coupling Code. Comput. Phys. Commun. 2014, 185, 676-683.

(52) Artyukhov, V. Ya.; Pomogaev, V. A. Three-Center Integrals of One-Electron Operator of a SpinOrbit Interaction. Russ. Phys. J. 2000, 43, 590-600.

(53) Pomogaev, V. A.; Artyukhov, V. Y. Spin-Orbital Interaction in Molecular Complexes of Naphthalene with Anthracene Derivatives. J. Appl. Spectrosc. 2001, 68, 251-258.

(54) Baryshnikov, G. V.; Valiev, R. R.; Karaush, N. N.; Minaeva, V. A.; Sinelnikov, A. N.; Pedersen, S. K.; Pittelkow, M.; Minaev, B. F.; Ågren, H. Benzoannelated Aza-, Oxa- and Azaoxa[8]Circulenes as Promising Blue Organic Emitters. Phys. Chem. Chem. Phys. 2016, 18, 28040-28051.

(55) Samanta, P. K.; Kim, D.; Coropceanu, V.; Brédas, J.-L. Up-Conversion Intersystem Crossing Rates in Organic Emitters for Thermally Activated Delayed Fluorescence: Impact of the Nature of Singlet vs Triplet Excited States. J. Am. Chem. Soc. 2017, 139, 4042-4051.

(56) Daza, M. C.; Doerr, M.; Salzmann, S.; Marian, C. M.; Thiel, W. Photophysics of Phenalenone: Quantum-Mechanical Investigation of Singlet-Triplet Intersystem Crossing. Phys. Chem. Chem. Phys. 2009, 11, 1688.

(57) Baryshnikov, G. V.; Valiev, R. R.; Minaev, B. F.; Ågren, H. Substituent-Sensitive Fluorescence of Sequentially N-Alkylated Tetrabenzotetraaza[8]Circulenes. New J. Chem. 2017, 41, 7621-7625.

(58) Valiev, R. R.; Cherepanov, V. N.; Baryshnikov, G. V.; Sundholm, D. First-Principles Method for Calculating the Rate Constants of Internal-Conversion and Intersystem-Crossing Transitions. Phys. Chem. Chem. Phys. 2018, 20, 6121-6133.

(59) Brédas, J.-L.; Beljonne, D.; Coropceanu, V.; Cornil, J. Charge-Transfer and Energy-Transfer Processes in $\pi$-Conjugated Oligomers and Polymers: A Molecular Picture. Chem. Rev. 2004, 104, 4971-5004. 
TOC Figure:

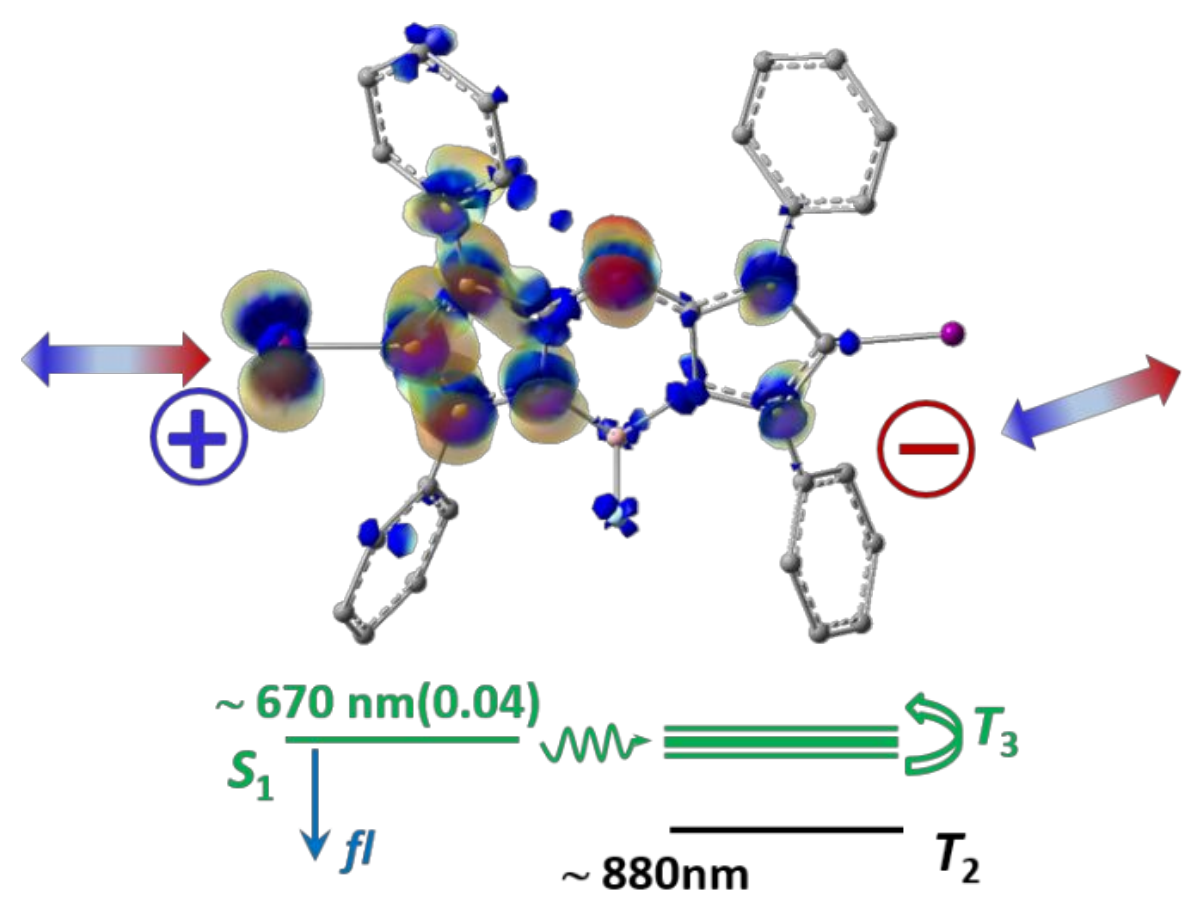

